\title{
Evaluación de un programa de monitoría de la calidad de los servicios otorgados por una Organización no Gubernamental
}

\author{
Alma Lucila Sauceda-Valenzuela, M. en C., ${ }^{(1)}$ Luis Durán-Arenas, Ph.D., (') \\ Bernardo Hernández, Ph.D. ${ }^{(2)}$
}

\begin{abstract}
Sauceda-Valenzuela AL, Durán-Arenas $L$, Hernández B.

Evaluación de un programa de monitoría de la calidad de los servicios otorgados por una Organización no Gubernamental. Salud Publica Mex 2000;42:422-430.
\end{abstract}

\section{Resumen}

Objetivo. Evaluar el impacto de un programa de monitoría de la calidad sobre la presencia de eventos centinela y las actitudes y conductas del personal ante la presencia de los mismos en una Organización no Gubernamental. Material y métodos. De acuerdo con un diseño cuasi-experimental del tipo antes y después para evaluar el efecto del programa de monitoría de la calidad se realizó este trabajo de 1998 a 1999, en 13 clínicas de una Organización no Gubernamental. Para la valoración de los cambios en actitudes y conductas se utilizó la comparación de diferencia de medias, y un análisis de varianza entre los grupos. Resultados. Los eventos centinela se redujeron de 32 detectados inicialmente a sólo dos en la evaluación. Se observaron diferencias de medias en el orden de +1.1 y +1.2 para actitudes y conductas en todos los eventos centinela $(p<0.05)$. Conclusiones. El sistema de monitoría de calidad tuvo el efecto buscado. Es importante destacar que después de la intervención predominaron las actitudes y conductas con propuestas de soluciones ante la ocurrencia de los eventos centinela.

Palabras clave: administración de la calidad total; calidad de la atención de salud; vigilancia de guardia; actitud del personal de salud; organizaciones no gubernamentales; México

\author{
Sauceda-Valenzuela AL, Durán-Arenas L, \\ Hernández B. \\ Evaluation of a quality-monitoring \\ program for services provided \\ by a Non-Governmental-Organization. \\ Salud Publica Mex 2000;42:422-430.
}

\begin{abstract}
Objective. To evaluate the effect of a quality-monitoring program on the occurrence of sentinel events and on attitudes and behaviors of personnel's responses in a Non-Governmental-Organization (NGO). Material and methods. Between 1998 and 1999, a quasi-experimental design of the before-after type was conducted in I 3 NGO clinics. Analysis of changes in attitudes and behaviors consisted in differences of means and analysis of variance between groups. Results. The number of sentinel events decreased from 32 events detected before the quality-monitoring program to only 2 after it. Attitudes and behaviors improved, with differences of means of $+I . I$ and $+1.2(p<0.05)$. Conclusions. The quality-monitoring program achieved the expected effects. It is noteworthy that attitudes and behaviors to prevent the occurrence of sentinel events were more prevalent after the intervention.
\end{abstract}

Key words: total quality management; quality of health care; sentinel surveillance; attitude of health personnel; nongovernmental organizations; Mexico

(I) Centro de Investigación en Sistemas de Salud, Instituto Nacional de Salud Pública (INSP), Cuernavaca, Morelos, México.

(2) Centro de Investigación en Salud Poblacional, INSP, Cuernavaca, Morelos, México.

Fecha de recibido: 2 de mayo de 2000 - Fecha de aprobado: 8 de agosto de 2000 Solicitud de sobretiros: Dr. Luis Durán Arenas. Centro de Investigación en Sistemas de Salud, Instituto Nacional de Salud Pública. Avenida Universidad 655, colonia Santa María Ahuacatitlán, 62508 Cuernavaca, Morelos, México. Correo electrónico: Iduran@insp3.insp.mx 
E 1 tema de la calidad de la atención en los servicios de salud tiene una amplia tradición en México y en países desarrollados. Sin embargo, a diferencia de otros países, en México existen muy pocas publicaciones en las que se describan los programas que se han implantado y menos aún los que notifiquen resultados de la evaluación de su efectividad e impacto. Es por esta razón que en este trabajo se presentan los efectos de un sistema de monitoría de la calidad sobre la manifestación de eventos centinela y las actitudes y conductas del personal ante dichos eventos en una Organización no Gubernamental (ONG) que presta servicios de salud reproductiva.

Esta ONG tiene en México más de 30 años de actividades y mantiene a lo largo de su existencia un gran interés por mejorar la calidad de la atención en sus servicios. Sin embargo, en la última década ha experimentado un crecimiento y diversificación (en términos de número de clínicas y tipos de servicios que presta), lo cual la ha puesto en una situación crítica en cuanto a su financiamiento y al control de la efectividad y eficiencia de los servicios que produce.

El programa de monitoría que aquí se presenta se diseñó como parte de la respuesta a los retos que enfrenta esta ONG, con base en la detección, corrección y prevención de eventos centinelas (EC), y en la respuesta a estos eventos por medio de estrategias de mejoramiento continuo de la calidad, así como por un sistema de supervisión capacitante implementado a nivel central de la ONG.

Para el desarrollo del programa se contó con la participación del personal relacionado con los procesos de atención médica en todas las clínicas participantes, incluyendo a administradores, coordinadores y proveedores médicos. De este grupo, se seleccionó la muestra en la que se evaluó el impacto del programa de intervención en términos del cambio de actitudes y conductas del personal.

La tradición de evaluación de la calidad de la atención médica tiene una larga historia. Su mayor desarrollo se ha dado en los Estados Unidos de América (EUA) donde las primeras acciones dirigidas hacia la calidad de la atención médica se desarrollaron principalmente en los hospitales. Por ejemplo, en los años veinte, el método de "resultados finales", de Codman, inició el interés por evaluar las consecuencias de las acciones médicas en términos de resultados finales. ${ }^{1}$

Posteriormente, en los años treinta, se enfatizó la elaboración de normas y estándares estructurales para establecer los fundamentos del proceso de la "buena atención médica", desarrollados por Lee y Jones. Todo ello condujo a la creación de la Comisión Conjunta de Acreditación de Hospitales, en 1951, ${ }^{2}$ un parteaguas que, después de un largo camino, logró la estandarización estructural de los hospitales en los EUA.

En los años setenta, el doctor Avedis Donabedian propuso una forma sistematizada para la definición y abordaje de los estudios sobre la evaluación de la calidad de la atención médica. En su obra destaca el análisis de la calidad mediante los enfoques de estructura, proceso y resultado; así como el análisis de la calidad por medio de sus tres dimensiones: calidad técnica, calidad interpersonal y comodidades. ${ }^{3,4}$

Los programas de mejora continua de la calidad provienen de la industria manufacturera y han sido adaptados en los últimos quince años en las industrias de servicios y en los últimos diez en el área de servicios de salud. El mejoramiento continuo de la calidad (MCC) se desarrolló a partir de las ideas de Shewart y Deming y se ha utilizado en las industrias japonesas y estadunidenses. ${ }^{5}$ Con base en el éxito en el mejoramiento de la calidad, las organizaciones internacionales de servicios de salud comenzaron a utilizar el MCC hacia finales de los años ochenta. Los ministerios de salud y las clínicas privadas y gubernamentales en más de 30 países comenzaron a emplear este enfoque innovador para adecuar sus programas. Muchos de los enfoques comparten elementos de esta técnica empresarial, pero algunas organizaciones de servicios de salud (por ejemplo, las que prestan servicios de salud reproductiva) han modificado el MCC formulando sus propios métodos y adaptándolos a sus necesidades. ${ }^{6,7}$

El MCC es equivalente a la gestión de la calidad total y consiste en un proceso, paso a paso, para el mejoramiento de la calidad utilizado por el personal considerando a la organización en forma completa e integral para introducir en la misma cambios en incrementos sucesivos. La gestión de la calidad total o mejoramiento continuo de la calidad implica, por lo tanto, planificar las mejoras, ejecutarlas y vigilar los resultados. ${ }^{8,9}$

Al utilizar dicho método, miembros del personal, con la ayuda inicial de consultores externos, examinan la prestación de los servicios y otros procesos para identificar los problemas y aplicar soluciones. De esta manera, los programas de mejoramiento continuo efectúan pequeñas mejoras continuas, en vez de grandes mejoras abruptas, a medida que la autoevaluación se convierte en una parte integral del sistema gerencial de las organizaciones.

La meta principal consiste en satisfacer las necesidades y expectativas del cliente, así, los miembros del personal trabajan en equipo para evaluar los procesos de prestación de los servicios de su organización, utilizando instrumentos tales como los diagramas de 
flujo y gráficos. Para corregir los problemas, el MCC se concentra en cambiar los procesos y no en culpar a los empleados. ${ }^{10}$

La primera experiencia en México de esfuerzos tendientes a mejorar la calidad de la atención se puede identificar cuando en el Hospital La Raza del Instituto Mexicano del Seguro Social (IMSS) se inició, en 1956, de manera extraoficial, la revisión de expedientes clínicos. ${ }^{11}$ Ello condujo, un año después, a la creación de la Comisión de Supervisión Médica del propio IMSS que después evolucionaría para convertirse, en 1972, en un área de evaluación médica; a partir de esa experiencia, en la década de los setenta, particularmente entre 1970 y 1976, la evaluación médica adquiere mayor relevancia y se inician desarrollos semejantes en el Instituto de Seguridad y Servicios Sociales de los Trabajadores del Estado (ISSSTE) y en el Sistema Nacional para el Desarrollo Integral de la Familia (DIF), con base en las auditorías médicas. Durante este periodo aparecen publicaciones sobre auditoría médica, evaluación de la relación médico-paciente y mortalidad como indicador de calidad.

En los primeros años de la década de los ochenta, continuó el interés por la calidad aun cuando no se identifica ningún fenómeno como el de la década anterior en el IMSS. En la segunda mitad de dicha década cobran fuerza nuevamente las acciones tendientes a mejorar la calidad, y se da la primera experiencia de mejoría de calidad como tal mediante la implantación de círculos de calidad en el Instituto Nacional de Perinatología, a partir de $1985 .{ }^{12}$ Los servicios médicos del entonces Departamento del Distrito Federal también introdujeron, a finales de 1980, los círculos de compromiso, como parte de un programa de reorganización y mejoramiento de la calidad en sus servicios, ${ }^{13} \mathrm{y}$ en el primer nivel de atención de los centros de salud de la Secretaría de la Salud se han dado experiencias en formación de círculos de calidad desde 1989.14

Los programas de MCC aplicados a la salud en México han tomado fuerza desde los ochenta y en el transcurso de la década pasada. Algunas experiencias de este tipo se han dado en hospitales grandes donde existen datos y recursos suficientes disponibles para la resolución de los problemas mediante las técnicas estadísticas que utiliza el MCC, sin embargo, existen pocas experiencias en el nivel primario y secundario de atención.

Recientemente, en la Secretaría de Salud, por medio de las acciones del Consejo de Salubridad General y del Consejo Nacional de Salud, se desarrollaron esfuerzos por normar la prestación de los servicios y se generó una iniciativa para la instrumentación de un sistema de información sobre calidad de la aten- ción que incluye por primera vez la propuesta de eventos centinela (EC) y de capacitación para la implantación de programas de mejora continua de la calidad. ${ }^{15}$

La detección de los EC como un programa que forma parte del MCC en los servicios de salud en el país tiene pocos antecedentes, sin embargo, se encuentran experiencias parecidas en la Secretaría de Salud para la monitoría y vigilancia de casos incidentes con VIH y la vigilancia epidemiológica de EC.

En este trabajo se emplean las siguientes definiciones de actitud y de conducta:

- "la actitud consiste en una disposición organizada a través de la experiencia, que ejerce una influencia directiva o dinámica sobre la conducta del individuo ante todos los objetos y situaciones con los que se relaciona"; $; 6$

- las actitudes son afirmaciones evaluativas -favorables o desfavorables- en relación con objetos, personas o hechos. Reflejan la forma en que una persona se siente acerca de algo. Uno de los investigadores de este tema, Thurstone, consideró que la actitud es "la suma total de las inclinaciones humanas, sentimientos, prejuicios, ideas preconcebidas, temores y amenazas", ${ }^{17,18} \mathrm{y}$

- la conducta es una acción realizada por el individuo ante una situación o contexto particular. ${ }^{19}$

Por su parte, Shijiriev describe tres componentes estructurales de la actitud:

1. Componente cognitivo. Es el componente informativo de las actitudes. Son las ideas o información que se tiene acerca del objeto de actitud, lo que se conoce del objeto de actitud, es el aspecto perceptivo.

2. Componente afectivo. Se refiere a los sentimientos y emociones que el objeto de actitud despierta en nosotros, lo que se siente acerca del objeto de actitud.

3. Componente conductual. Indica el comportamiento del individuo respecto al objeto de actitud. Es el sistema de acciones exteriormente observable, mediante el que se expresa la actitud, por lo cual se posibilita evaluar su existencia. Este componente se puede conocer mediante el análisis de la conducta, la actividad y expresiones verbales indicativas de conducta. ${ }^{20}$

Varias características son comunes a toda clase de actitudes: se aprenden o adquieren en el curso de la experiencia diaria, no son innatas, ya que desde la in- 
fancia se van adquiriendo tradiciones, creencias, opiniones y conocimientos que contribuyen a crearlas; no siempre están relacionadas con la educación o la inteligencia de la persona ni con los hechos de la situación; suelen tener más fundamentos emocionales que racionales; se pueden fortalecer, modificar o eliminar, es decir, son flexibles, aunque todo cambio requiere de tiempo y dirección hábil.

Las actitudes reciben la influencia de elementos tales como: el ambiente físico y social de la persona, las experiencias pasadas, los deseos y metas y los aspectos fisiológicos del individuo. La actitud que tenga una persona hacia un valor en particular se puede determinar por una opinión verbal o por la conducta de la persona en relación con el valor. Los individuos suelen tener actitudes favorables hacia las cosas que resultan en la satisfacción de sus aspiraciones y actitudes desfavorables hacia aquellas cosas que impiden la satisfacción de sus necesidades.

Las actitudes forman la estructura fundamental de la conducta organizacional de un trabajador o de un grupo de ellos, y deben ser de gran importancia para los directivos en instituciones hospitalarias. Como las actitudes son recopilaciones de información y de experiencias del pasado a las que el individuo ha estado expuesto son difíciles de predecir. Por lo cual es necesario evaluarlas con regularidad para que los directivos dirijan a sus trabajadores de una forma adecuada.

En general, las actitudes y las conductas tienen una relación estrecha con la calidad, porque para que un servicio (de cualquier índole) se otorgue con calidad se necesitan actitudes y conductas dirigidas a la mejora continua.

Hace más de medio siglo, Lee y Jones reconocieron en su exploración del concepto de calidad que ésta significa conformidad con normas y que dichas normas no son absolutas sino que varían de una sociedad a otra. La definición clásica contemporánea de la calidad de la atención en salud ha sido desarrollada por Donabedian. De acuerdo con él, la calidad es una propiedad de la atención médica que puede ser obtenida en diversos grados. Esa propiedad se puede definir como la obtención de los mayores beneficios posibles de la atención médica con los menores riesgos para el paciente, en donde los mayores beneficios posibles se definen, a su vez, en función de lo alcanzable de acuerdo con los recursos con los que se cuenta para proporcionar la atención y de acuerdo con los valores sociales imperantes. ${ }^{21}$

Por otra parte, los expertos del MCC, como Deming, Crosby y Juran, la definen como satisfacer las necesidades y las expectativas de los clientes -en los servicios de salud, los clientes son los usuarios de los servicios. La medición de la calidad, desde este último punto de vista, significa aparentemente cuantificar algo que es inherentemente subjetivo. Si bien los profesionales de la atención de la salud y los usuarios o clientes tienden a ver la calidad desde perspectivas diferentes, ambos puntos de vista son válidos e importantes para el desarrollo del MCC. ${ }^{22}$

Por lo anterior es que en este trabajo se ha generado la siguiente definición operativa de la calidad: la obtención de los mayores beneficios posibles dada la puesta en práctica de los componentes del sistema de monitoría de la calidad, con los menores riesgos para el utilizador de los servicios de atención en esta ONG, donde se definen los mayores beneficios posibles como los aumentos alcanzables de acuerdo con los recursos y con los valores sociales de la ONG.

\section{Material y métodos}

Este estudio, realizado entre 1998 y 1999 en clínicas de una ONG, sigue un diseño cuasi-experimental del tipo antes y después, ya que se partió de una evaluación de actitudes y conductas previa al programa de intervención de MCC y, una vez finalizado éste, se llevó a cabo una segunda evaluación utilizando el mismo cuestionario; cada clínica fue su propio control.

Se eligieron 13 clínicas de las 14 con que cuenta esta ONG; sin embargo, sólo se evaluaron 10 que representan $71 \%$ del universo de estudio; se descartaron tres clínicas porque el personal fue removido de su puesto y no fue posible hacer en ellos la medición después de la intervención. Estas 10 clínicas evaluadas se encuentran en los estados de Nuevo León, Jalisco, Sonora, Michoacán, Tamaulipas, Veracruz, Estado de México y Distrito Federal.

Todo el personal de las clínicas participó en el programa de mejoría, lo cual es la norma en la instrumentación de programas de MCC. El personal que se seleccionó para la evaluación fue: el coordinador médico de cada clínica, el administrador de cada clínica $\mathrm{y}$, aleatoriamente, a un médico por clínica, dando un total de 30 informantes.

La intervención en este estudio incluyó: el sistema de monitoría de EC, el uso de la estrategia de tableros de calidad como la base para la resolución de los problemas detectados y los cambios organizacionales en la ONG para dar seguimiento a estas acciones.

La presencia de un EC debe generar una investigación sistemática de las causas relacionadas con su aparición, y debe ser seguida por una acción correctiva inmediata con el fin de prevenir la ocurrencia futura de un EC similar en la misma unidad. Por esta razón, el sistema de detección de estos eventos es con- 
siderado en este estudio como un mecanismo de monitoreo que dispara el proceso de mejora continua de la calidad. Los EC deben ser controlados antes de intentar abordar la variabilidad común de los procesos en un ambiente cotidiano, una vez logrado, el MCC se puede concentrar en oportunidades de mejora de una manera prospectiva.

El sistema de monitoría de EC incluye tres instrumentos: a) informes inmediatos, que deberán ser enviados antes de 24 horas de la aparición de un EC; b) indagación inicial, una vez que se tiene un informe inmediato se deberá llevar a cabo un análisis preliminar de las causas del problema siguiendo la estrategia del tablero de calidad, el informe deberá ser enviado en el transcurso de las dos semanas subsecuentes a la aparición del evento centinela, y c) evaluación concluyente, una vez aplicada la estrategia del tablero de calidad se deberá enviar la evaluación concluyente que tiene el objetivo de compartir las soluciones a los problemas analizados en toda la organización.

El programa de MCC que utilizó esta ONG se basa en la estrategia del tablero de calidad como la herramienta de trabajo principal para la resolución y prevención de EC y para los proyectos de mejoría. Asimismo, el tablero de mejoría continua se define como un proceso sistemático de abordaje de oportunidades de mejora que usa la estrategia de solución de problemas a partir de datos no rutinarios, cuya estructura ayuda a ilustrar las etapas que debe seguir un equipo en el proceso de mejoría y brinda una forma estandarizada de comunicación en la formación, evolución y desempeño de los equipos. ${ }^{22}$

Finalmente, se diseñó, en conjunción con las autoridades de la ONG, un sistema de supervisión capacitante que fue aplicado para supervisar el avance de las acciones de mejora.

En los servicios de salud, al igual que en cualquier tipo de servicios, la calidad está estrechamente relacionada con las actitudes y conductas de los trabajadores. Los servicios están basados en una relación cara a cara entre el usuario y el proveedor del servicio. La actitud y la conducta del proveedor determinan en gran parte la calidad de dichos servicios. Es por esto que en este estudio se evaluaron las actitudes y conductas como proxis de la efectividad de la intervención.

Se seleccionó una clínica para la prueba piloto del sistema de detección de EC y MCC, en la cual se dio una capacitación de cuatro días, con tres visitas subsecuentes de asesoría. Posteriormente, se definieron las 12 clínicas que se unirían a este estudio y con ayuda del personal participante del Instituto Nacional de
Salud Pública (INSP) se realizó la capacitación con base en los resultados de la prueba piloto, esta capacitación duró tres días y previa a ella se levantó la medición basal del estudio.

Las tareas a seguir por el personal capacitado una vez en su trabajo cotidiano fue la conformación de equipos de trabajo o de mejoría que funcionarían de la siguiente forma: una vez identificado un EC se haría su notificación al administrador de la clínica, el cual sería responsable de reportar este evento a las oficinas centrales de la ONG para su conocimiento; el equipo de mejora conformado sería el encargado de la investigación, que comenzaría a sesionar lo antes posible en su lugar de trabajo, utilizando la metodología del tablero de mejoría continua de la calidad. Posteriormente, se indagaría en los registros históricos existentes en la unidad todos los datos posibles relacionados con la ocurrencia y la frecuencia de factores vinculados con el EC, identificados a partir del diagrama de flujo del proceso; en un máximo de cinco días hábiles, a partir de la fecha de ocurrencia del evento, el equipo de mejora habría completado las etapas arriba descritas y enviaría un reporte con esta información como anexo del Formato de indagación inicial a las oficinas centrales de la ONG, este formato incluye elementos que profundizan la información contenida en la notificación inmediata.

En un plazo no mayor a 30 días, a partir de la fecha de ocurrencia del evento, el equipo de mejora debería entregar un reporte por escrito que contenga los productos obtenidos durante el trabajo desarrollado en las primeras cuatro etapas del tablero de mejora al personal encargado del programa en las oficinas centrales de la ONG. Sin embargo, el equipo continuaría reportando periódicamente la monitoría que lleve al cabo de los resultados obtenidos y del proceso de estandarización de las medidas correctivas.

A los dos meses de iniciado el programa de intervención se procedió a realizar las visitas de supervisión capacitante a las clínicas para valorar el grado de avance del programa de detección de EC. En estas visitas se corroboró el desarrollo del programa en algunas clínicas, y se asesoró nuevamente en las que presentaban fallas o dudas respecto al programa y al trabajo del tablero de mejoría continua de la calidad.

Finalmente, después de cuatro meses, se levantó el segundo cuestionario que permitió evaluar el impacto de la intervención en las actitudes y conductas del personal involucrado en los procesos clínicos y administrativos de atención de las clínicas participantes. 
Para la evaluación del efecto de la intervención se diseñó un cuestionario que se estructuró de la siguiente manera:

1. Datos de identificación con características individuales del personal (edad, sexo, profesión, cargo) y características de las clínicas (tipo de clínica).

2. Presencia de los eventos centinela en las clínicas.

3. Actitud actual o futura de las personas entrevistadas ante la presencia de cada uno de los eventos centinela.

4. Conducta actual o futura de las personas entrevistadas ante la presencia de cada uno de los eventos centinela.

5. Percepción de las conductas de otras personas ante la presencia de un evento centinela, desde el punto de vista de la persona entrevistada.

La evaluación de la presencia del EC es una variable dicotómica que permite clasificar la presencia o no de cada uno de ellos. El objetivo de esta medición es ver la reducción de la presencia de los EC seleccionados como consecuencia de la intervención. Estos eventos fueron definidos como indicadores que cuantifican la presencia de eventos con consecuencias negativas para la organización. Esta definición es una ampliación de la definición original de Rutstein, de tal manera que se aplique a eventos negativos, diferentes a la muerte y la morbilidad evitables. ${ }^{23}$

Los EC para el monitoreo de la calidad que se estudiaron en esta intervención fueron los siguientes:

1. Complicaciones quirúrgicas. Toda contingencia en el estado de salud de cualquier paciente sometido a un acto quirúrgico programado en el Centro de Servicios Médicos de la ONG, que requiera del traslado inmediato a una unidad hospitalaria o de la utilización de maniobras o aplicación de medicamentos de urgencia, durante el acto quirúrgico y hasta 48 horas posteriores a la ejecución de éste.

2. Demanda insatisfecha. Toda queja expresada en primera persona y de manera directa a un miembro de los programas comunitarios de la ONG, en relación con la negación de un servicio, mal trato en su otorgamiento, tiempo de espera mayor a 45 minutos, abuso o violación a la intimidad o pudor de la paciente, ocurridos durante el proceso de atención en el Centro de Servicios Médicos durante las cuatro semanas previas a la queja.

3. Problemas intrahospitalarios. Toda alta voluntaria cuyo origen se encuentre en una queja específica por alguna deficiencia en el servicio, agravamiento del estado de salud del paciente o la adquisición de una entidad patológica durante su estancia hospitalaria en el Centro de Servicios Médicos.

4. Quejas en la consulta externa. Todo usuario que solicite y abandone el Centro de Servicios Médicos sin recibir ninguna atención, después de $45 \mathrm{mi}$ nutos de espera durante el día o 15 minutos durante la noche.

Por su parte, para las variables de actitudes y conductas es importante clarificar que dado el costo involucrado y la imposibilidad práctica de evaluar actitudes y conductas ante la presencia de un EC particular, lo que se interrogó fue lo que el actor realizaría ante la presencia de un EC y se preguntó sobre cada uno de los eventos definidos en el estudio.

Las preguntas de actitudes y conductas se operacionalizaron de la siguiente manera:

- actitud: toda opinión o percepción manifestada por los administradores, coordinadores y proveedores de servicios médicos en relación con los EC, y

- conducta: todo comportamiento manifestado por los administradores, coordinadores y proveedores de servicios médicos en relación con los EC.

Se hizo análisis de contenido en las respuestas a las preguntas de actitud y conducta y se crearon variables ordinales, procediendo de la siguiente manera:

1. Las actitudes se clasificaron de nivel negativo a positivo como: quejas, indiferencia, posiciones personales, cooperación o ayuda, propuestas de soluciones.

2. Las conductas se clasificaron de nivel negativo a positivo como: entorpecimiento o retraso en el trabajo, quejas, indiferencia, cooperación o ayuda, propuestas de soluciones.

Se consideró como propuesta de solución a toda actitud o conducta orientada a dar solución viable y benéfica a cualquier EC; en relación con la operacionalización de cooperación o ayuda ésta se definió como toda actitud o conducta enfocada a auxiliar o apoyar la mejor solución ante la presencia de cualquier EC; se consideró a la indiferencia como toda actitud o conducta pasiva que no muestre ningún interés en solucionar o prevenir los EC; asimismo, las posiciones personales se consideraron como toda actitud que haga alusión sólo a cuestiones personales ante la presencia de los EC, por ejemplo, es mi responsabilidad, yo soy una profesionista, es un compromiso, etcétera; en relación con las quejas éstas incluyeron toda ac- 
titud o conducta que culpe a otras personas o a la institución de la ocurrencia de los EC; así, se consideró entorpecimiento o retraso hacia el trabajo toda conducta en contra de los procesos de trabajo involucrados en los EC.

Esta metodología sigue los principios para la construcción de escalas del tipo Likert ${ }^{24}$ que produce variables ordinales de cinco puntos. Se calcularon promedios antes y después de la intervención así como la diferencia de medias para evaluar el impacto de dicha intervención. Se aplicó la prueba de $t$ de Student para datos pareados para valorar la significancia estadística de la diferencia de medias, para evaluar la magnitud de los cambios en las actitudes y conductas hacia los EC antes y después del programa de intervención; también se realizó un análisis de varianza de medidas repetidas con el fin de valorar la variación en los cambios entre los grupos de menor riesgo $v s$ los grupos de mayor riesgo.

\section{Resultados}

Las clínicas del estudio no eran homogéneas. Cuatro de las 10 clínicas del estudio daban atención exclusiva de consulta externa y urgencias con capacidad para cirugía ambulatoria, mientras que las seis restantes contaban con consulta externa, urgencias y áreas de hospitalización. Las clínicas con hospitalización tenían en promedio seis camas censables por clínica, y los servicios que se prestan son fundamentalmente atención del parto y cirugía de corta estancia (incluyendo vasectomía). La clínica con mayor número de personal fue una de consulta externa, ubicada en un área metropolitana contando con un total de 21 prestadores de servicios, mientras que la clínica con menor número de trabajadores fue una de reciente fundación ubicada en provincia que contaba tan sólo con cinco prestadores médicos.

En la medición basal se identificaron $32 \mathrm{EC}$ en las clínicas de estudio. De éstos, predominaron las menciones sobre las demandas insatisfechas (se observó en todas las clínicas), las quejas en consulta externa (se observó en 9 de 10 clínicas) y las complicaciones quirúrgicas (en 8 de 10 clínicas). Al momento de la evaluación final se observó una franca reducción en la presencia de EC; por ejemplo, las complicaciones quirúrgicas no se observaron al final de la evaluación. Las demandas insatisfechas se redujeron en $90 \%$; las quejas en consulta externa en $89 \%$, aunque en dos clínicas persistía el problema al momento de la segunda evaluación.

Los dos EC que persistieron fueron las quejas en consulta externa y las demandas insatisfechas, ambos se reportaron en dos clínicas que dan consulta externa. Dichas clínicas son diferentes a las demás ya que cuentan con una gran demanda de atención, éste puede ser el motivo por el cual no se pudo eliminar su presencia de la misma forma que en las otras clínicas en la segunda medición.

Para examinar la magnitud de las diferencias antes y después de la intervención se aplicó una prueba de diferencia de medias (cuadro I). En términos generales, se observó una mejoría en relación con las actitudes y conductas ante la presencia de los EC por parte del personal participante en el programa. En general, la actitud encontrada era inicialmente de posiciones personales e indiferencia y la conducta era de tratar de esconder el evento e indiferencia. En la medición final la actitud cambió y se mantenía el personal cooperativo ante la posibilidad de un EC y la conducta era la de utilizar las herramientas de mejora de la calidad para resolver el problema. Por ejemplo, en complicaciones quirúrgicas se observó una diferencia de medias de $+1.1,+1.2,+1.1$ para actitudes, conducta $y$ conductas de otro personal, respectivamente $(p<0.05)$. Se observó el mismo efecto en las demandas insatisfechas, los problemas intrahospitalarios y las quejas en consulta externa, en todos los casos con valores de $p<0.05$.

En el cuadro II se presentan los resultados del análisis de diferencia de medias de las actitudes y conductas hacia los EC, desglosado por el tipo de personal participante en las clínicas. Se encontró que se mantenían las diferencias estadísticamente significativas en los diferentes puestos del personal de estudio; esto sugiere que el cambio en las actitudes y conduc-

\section{Cuadro I \\ Diferencia de medias de las actitudes Y CONDUCTAS HACIA LOS EVENTOS CENTINELA. TODOS LOS ENTREVISTADOS}

\begin{tabular}{|c|c|c|c|c|c|c|}
\hline \multirow{5}{*}{$\begin{array}{l}\text { Eventos centinela } \\
\text { Complicaciones quirúrgicas }\end{array}$} & \multicolumn{6}{|c|}{ Personal de las clínicas } \\
\hline & \multirow{3}{*}{\multicolumn{2}{|c|}{$\begin{array}{c}\text { Actitud } \\
\text { Media } \\
\text { Antes-después }\end{array}$}} & \multirow{3}{*}{\multicolumn{2}{|c|}{$\begin{array}{c}\text { Conducta } \\
\text { individual } \\
\text { Media } \\
\text { Antes-después }\end{array}$}} & \multirow{3}{*}{\multicolumn{2}{|c|}{$\begin{array}{l}\text { Conductas de } \\
\text { otro personal } \\
\text { Media } \\
\text { Antes-después }\end{array}$}} \\
\hline & & & & & & \\
\hline & & & & & & \\
\hline & 3.1 & $4.2^{*}$ & 3.5 & $4.7^{*}$ & 3.2 & $4.3^{*}$ \\
\hline Demanda insatisfecha & 3.4 & $4.6^{*}$ & 3.5 & $4.8^{*}$ & 3.5 & $4.3^{*}$ \\
\hline Problemas intrahospitalarios & 3.6 & $4.3^{*}$ & 3.6 & $4.7^{*}$ & 3.1 & $4 . I^{*}$ \\
\hline Quejas en consulta externa & 3.0 & $4.4^{*}$ & 3.6 & $4.8^{*}$ & 3.5 & $4.5^{*}$ \\
\hline
\end{tabular}

Fuente: Encuesta a las clínicas de la Organización no Gubernamental, México, 1998-1999

salud pública de méxico / vol.42, no.5, septiembre-octubre de 2000 


\section{Cuadro II \\ Diferencia de medias de las actitudes Y CONDUCTAS HACIA LOS EVENTOS CENTINELA, SEGÚN PUESTO DEL PERSONAL DE ESTUDIO}

\begin{tabular}{|c|c|c|c|c|c|c|}
\hline \multirow[t]{2}{*}{ Eventos centinela } & $\begin{array}{r}M \\
\text { Antes- }\end{array}$ & $\begin{array}{l}\text { dia } \\
\text { espués }\end{array}$ & \multicolumn{2}{|c|}{$\begin{array}{l}\text { Conducta } \\
\text { individual }\end{array}$} & $\begin{array}{l}\text { Conductas de } \\
\text { otro personal }\end{array}$ & $\begin{array}{l}\text { ctas de } \\
\text { ersonal } \\
\text { edia } \\
\text { después }\end{array}$ \\
\hline & \multicolumn{6}{|c|}{ Administradores $n=10$} \\
\hline Complicaciones quirúrgicas & 3.0 & $3.8^{*}$ & 3.9 & $4.6^{*}$ & 3.1 & $4.1^{*}$ \\
\hline Demanda insatisfecha & 3.6 & $4.5^{*}$ & 3.5 & $5.0^{*}$ & 3.5 & $4.4^{*}$ \\
\hline Problemas intrahospitalarios & 4.0 & $4.5^{*}$ & 3.6 & $4.7^{*}$ & 3.3 & $4.2^{*}$ \\
\hline \multirow[t]{2}{*}{ Quejas en consulta externa } & 3.4 & $4.6 *$ & 3.6 & $4.9^{*}$ & 3.4 & $4.7^{*}$ \\
\hline & \multicolumn{6}{|c|}{ Coordinadores médicos $n=10$} \\
\hline Complicaciones quirúrgicas & 3.4 & $4.6 *$ & 3.6 & $4.7^{*}$ & 3.3 & $4.3^{*}$ \\
\hline Demanda insatisfecha & 3.3 & $4.6^{*}$ & 3.5 & $4.7^{*}$ & 3.4 & $4.4^{*}$ \\
\hline Problemas intrahospitalarios & 3.4 & $4.2^{*}$ & 3.5 & $4.8^{*}$ & 2.8 & $4.0^{*}$ \\
\hline \multirow[t]{2}{*}{ Quejas en consulta externa } & 3.0 & $4.2^{*}$ & 3.7 & $4.6^{*}$ & 3.6 & $4.5^{*}$ \\
\hline & \multicolumn{6}{|c|}{ Proveedores médicos $n=10$} \\
\hline Complicaciones quirúrgicas & 2.9 & $4.2 *$ & 3.0 & $4.7^{*}$ & 3.3 & $4.4^{*}$ \\
\hline Demanda insatisfecha & 3.3 & $4.8^{*}$ & 3.5 & $4.8^{*}$ & 3.6 & 4.2 \\
\hline Problemas intrahospitalarios & 3.3 & $4.2^{*}$ & 3.8 & $4.7^{*}$ & 3.2 & $4.0^{*}$ \\
\hline Quejas en consulta externa & 2.7 & $4.3^{*}$ & 3.5 & $4.8^{*}$ & 3.4 & $4.4^{*}$ \\
\hline
\end{tabular}

Fuente: Encuesta a las clínicas de la Organización no Gubernamental, México, 1998-1999

tas posterior al programa de intervención fue general en todo el personal.

En el análisis de varianza de medidas repetidas se compararon los cambios de actitud y conducta antesdespués hacia los cuatro EC por parte de los administradores, coordinadores y proveedores médicos; se observó una variación significativa en los cambios de las actitudes manifestada por los administradores y coordinadores médicos hacia los eventos de complicaciones quirúrgicas, problemas intrahospitalarios y quejas en consulta externa, esta variación también se observó en las conductas de otro personal manifestada por los proveedores médicos hacia las quejas en consulta externa, en todos estos casos el valor de $p$ fue $<0.05$.

La variación de los cambios en las actitudes antes-después fue mayor en las personas que ocupan puestos directivos y en teoría de menor riesgo (administradores y coordinadores médicos) en comparación con las personas que ocupan puestos más operativos teóricamente de mayor riesgo (proveedores médicos). A diferencia de las conductas, donde no se observaron variaciones en los cambios de un grupo a otro.

\section{Discusión}

En este estudio fue posible evaluar el efecto a corto plazo de la implantación de un sistema de monitoría de la calidad que incluye un programa de MCC. De acuerdo con los resultados, el sistema tuvo el efecto buscado, no sólo en la prevención de la aparición de nuevos EC sino también en un cambio de las actitudes y conductas del personal directivo, prestador de servicios y administrativo. La intervención que se implementó fue utilizada adecuadamente, y permitió no sólo monitorear la calidad de la atención sino que sirvió para capacitar y evaluar el desempeño de la clínicas.

La virtual desaparición de los EC estudiados muestra el potencial de este tipo de acciones. Esto se correlacionó con un cambio importante y significativo en las actitudes y conductas del personal de las clínicas. Aunque no se sabe qué ocurre primero, se sugiere que el cambio en actitudes y conductas permitió que esta organización respondiera de manera más efectiva a los EC y transmitiera estas experiencias no sólo dentro de las clínicas sino a toda la organización.

Los resultados sugieren la existencia de un cambio en las actitudes y conductas del personal como consecuencia del programa de MCC, sin embargo, es importante eliminar otras causas potenciales del cambio en actitudes y conductas. Por ejemplo, existe la posibilidad de que la presencia del personal responsable del programa pudo haber causado un efecto Hawtorne. ${ }^{25}$ Sin embargo, la magnitud del cambio confirma la existencia de un efecto positivo en las clínicas. Es importante destacar que no sólo se evidenció un cambio general de actitudes sino que predominaron las actitudes y conductas con propuestas de solución o de cooperación o ayuda ante la ocurrencia de los EC en la medición final.

En resumen, los resultados de este estudio permiten sustentar la relevancia de este tipo de intervenciones gerenciales para la mejora de la calidad. Intervenciones sencillas como ésta, que permiten utilizar la información para la toma de decisiones, podrían ser implantadas con éxito en organizaciones similares y en organizaciones públicas, ya que implican un bajo costo y un potencial de mejora importante.

En términos de su relevancia para el desarrollo de programas de MCC, este estudio sugiere que la sistematización del proceso de monitoría aunado a la implantación de programas de MCC incrementa el efecto. 
No basta con la disposición a participar en un proceso de mejora, el impulso que da el fijar EC y a partir de ellos iniciar acciones resultó altamente productivo.

Por otra parte, el estudio también aporta ideas acerca de los mecanismos por medio de los cuales los programas de MCC pueden expresar su impacto. El cambio de actitudes y sus consecuentes conductas son aspectos que se mencionan pero rara vez se evalúan. En el presente estudio la motivación lograda y el cambio de actitudes señalan la necesidad de estudiar a fondo los mecanismos que garanticen que estos cambios se den no sólo a corto plazo sino que se mantengan a mediano y largo plazo.

Finalmente, es importante llamar la atención hacia la evaluación de intervenciones para mejorar la calidad. Existen muchos programas en diferentes instituciones, hospitales y ONG que no se conocen, o si se conocen no han sido evaluadas. Es importante para el desarrollo del campo fomentar la publicación de estas experiencias. Esperamos que ésta sea una contribución en ese sentido y que muchas otras sean compartidas.

\section{Agradecimientos}

Se agradece a la doctora Blanca Pelcastre Villafuerte y a la maestra Carolina González Schlenker por los comentarios para la realización de este trabajo.

\section{Referencias}

I. Kazandjian VA. From mythology to epidemiology:The historic roots of continuous quality improvement. En: Kazandjian VA, ed. The effectiveness of CQI in health care. Stories from a global perspective. Milwakee (Wis): ASQC Quality Press, 1997: I-9.

2. Ruelas-Barajas E. Hacia una estrategia de garantía de calidad. De los conceptos a las acciones. Salud Publica Mex 1991;34:29-45.

3. Donabedian A. Twenty years of research on the quality of medical care. 1964-1984. Eval Health Prof 1985;8:243-265.

4. Donabedian A. The definition of quality and approaches to its assessment. Ann Arbor (Mich): Health Administration Press, 1980;vol. I.
5. Rosander AC. Los catorce puntos de Deming aplicados a los servicios. Ann Arbor (Mich): Díaz de Santos, 1994:1-7.

6. Network en español. Fam Health Int 1993;8: I-33.

7. Jain A, Bruce J, Mensch B. Setting standards of quality in family planning programs. Stud Fam Plann 1992; 23(6):392-95

8. Alvear-Sevilla C. Calidad Total II:Aseguramiento y mejora continua. México, D.F.Limusa, 1999:167.

9. Juran JM. Juran on planning for quality. Nueva York: Free Press, 1988.

10. Nicolas DD, Winter D, Crespind J, Boukar AM. Results of CQI in Africa:The Niger experience. En: Kazandjian VA, ed. The effectiveness of $\mathrm{CQI}$ in health care: Stories from a global perspective. Milwakee (Wis): ASQC Quality Press, 1997:258.

II.Aguirre-Gas H.Administración de la calidad de la atención médica. Rev Méd Inst Mex Seguro Soc 1997;35:257-264.

12. Ruelas-Barajas E, Reyes-Zapata H, Zurita-Garza B, Vidal-Pineda LM, Karchmer $S$. Círculos de calidad como estrategia de un programa de garantía de calidad de la atención médica en el Instituto Nacional de Perinatología. Salud Publica Mex 1990;32:207-220.

13. Laguna-Calderón J, Salinas-Oviedo C. Mejoría de la calidad de los servicios médicos del Departamento del Distrito Federal. Salud Publica Mex 1990;32:221-224.

14. Reyes-Zapata H, Meléndez-Colindres VM, Vidal-Pineda LM. Programa de garantía de calidad en el primer nivel de atención. Salud Publica Mex 1990;32:232-242.

15. Durán-Arenas L, Cruz-Rivero C, Fernández-Cantón S, Sánchez-Rodríguez $R$, Franco $F$,Wong-Luna $R$ et al.The development of a quality information system: A case study of Mexico. Health Policy Plann 1998;13(4): 446-458.

16. Casales JC. Psicología social. La Habana: Editorial de las Ciencias Sociales, 1989:160-186.

17. Robbins SP. Comportamiento organizacional: teoría y práctica. 7a. edición. México, D.F.: Prentice Hall, 1996:180-190.

18. Morgan WJ Jr. Administración de personal de instituciones hospitalarias. México, D.F:: Limusa, 1983:143-148.

19. Babbie E. The practice of social research. 4a. edición. Belmont (CA): Wadsworth Publishing, 1986:53.

20. Morales JF, Olza M. Psicología social y trabajo social. Madrid: Mc Graw Hill, 1998.

21. Donabedian A. Quality assurance in our health care system. Am Coll Util Rev Physi 1986; 1:6-I2.

22. Durán-Arenas L, Carreón J,Vallejo M, Sánchez-Rodríguez R. The Continuous Quality Improvement Program in the Mexican Secretary of Health. En: Kazandjian VA, ed. The effectiveness of CQI in health care. Milwakee (Wis): Asoc Quality Press, 1997:I I-48.

23. Rutstein DD, Berenberg W, Chaliners TC. Measuring the quality of medical care. New England J Med 1976;294:582-588.

24. Namakforoosh MN. Metodología de la investigación. México, D.F.: Limusa, 1998:24|-246.

25. Perrow C. Sociología de las organizaciones. 3a. edición. Madrid: Mc Graw Hill, 1993:96-104. 